\title{
SISTEMA INFORMÁTICO PARA LA PLANIFICACIÓN DE LA PRODUCCIÓN EN MICRO Y PEQUEÑAS EMPRESAS DE CONFECCIONES
}

\author{
MARÍA del Rocío ÁvILA GONZALES DE TRINIDAD* \\ https://orcid.org/0000-0002-8672-1612 \\ Universidad de Lima
}

Recibido: 1 de agosto del 2020 / Aprobado: 10 de septiembre del 2020

doi: https://doi.org/10.26439/ing.ind2021.n40.5146

RESUMEN: El sistema informático para la planificación de la producción determina qué y cuánto producir para cumplir con las metas propuestas por las mypes (micro y pequeñas empresas) de confecciones; esto es, satisfacer la demanda a tiempo y obtener la máxima utilidad. Utilizando el enfoque cuantitativo, se aplicó un modelo de programación matemática de optimización por metas y la metodología Scrum. Asimismo, se utilizó el software MS Excel, tanto para resolver el modelo matemático como para las bases de datos. Las interfaces para el ingreso de los datos y para el reporte solución se desarrollaron en la plataforma Java SE. El sistema informático se instaló en dos mypes, en ambas se incrementó la productividad y fue aceptado por su fácil uso y rápida adaptación. La llegada de la pandemia no permitió la instalación en otras mypes.

PALABRAS CLAVE: sistema informático / planificación de la producción / mypes

de confecciones / programación por metas

\section{A COMPUTER SYSTEM FOR PRODUCTION PLANNING IN CLOTHING SMALL ENTERPRISES AND MICROENTERPRISES}

ABSTRACT: The computer system for production planning determines what and how much to produce in order to achieve the goals set by clothing small and medium enterprises (SMEs); that is, to meet the demands on time and get the maximum benefit. Using a quantitative approach, a mathematical programming model for goal optimization and the Scrum framework were implemented in the study. In addition, MS Excel software was used for both the mathematical model and databases. The interfaces for data entry and reporting solution were developed in the Java SE platform. The computer system was installed in two SMEs: It increased productivity in both of them and was accepted for its ease of use and quick adaptation. The pandemic did not allow the installation of the computer system in other SMEs.

KEYWORDS: computer system / production planning / clothing SMEs / goal programming

*Correo electrónico: mavila@ulima.edu.pe 


\section{INTRODUCCIÓN}

En el Perú existe un importante sector productivo conformado por micro y pequeñas empresas (mypes) de confecciones que han crecido, principalmente, en un ambiente de informalidad (Cuatro de cada cinco mypes son informales: ¿es suficiente reducir el IGV?, 25 de agosto de 2016), superando toda expectativa y convirtiéndose en motores de un gran dinamismo comercial, sin embargo, no ha sido orientado por un desarrollo estratégico empresarial.

En general, las mypes son dirigidas empíricamente por pequeños empresarios que adquieren sus habilidades basados en la experiencia y en el conocimiento práctico, en la mayoría de los casos, observando a sus padres, guías o maestros. En los últimos años, este panorama ha cambiado, el 39,6\% de los participantes manifestaron haber concluido sus estudios universitarios, mientras que el 24,2 \% tenía secundaria completa (Instituto Nacional de Estadística e Informática, 2011).

En general, las mypes de confecciones presentan una baja utilización de los recursos ofrecidos por las tecnologías de la información y la comunicación al planificar la producción y mejorar la productividad. El porcentaje de mypes que optaron por implementar tecnologías para mejorar sus procesos productivos o servicios fue del 15,7\%; los valores más altos del indicador se encuentran en Cuzco (37,8 \%), Ayacucho (35\%), Huancayo $(28,8 \%)$ y Lima y Callao (15,3 \%) (Instituto Nacional de Estadística e Informática, 2011).

En la actualidad, los dueños de las mypes o empleados de confianza, generalmente, determinan, entre varios modelos de prendas, qué y cuánto producir, basándose, únicamente, en su experiencia y sentido común, sin aprovechar los métodos de optimización que se aplican en un sistema de planeamiento de la producción.

Es de vital importancia una producción variada que responda a la demanda del mercado, que cumpla los plazos de entrega, aproveche al máximo sus recursos, use toda la capacidad de producción (evitando tiempos improductivos) y obtenga una óptima utilidad a fin de lograr una mayor productividad, esto con el fin de que las mypes de confecciones deben competir con las prendas de vestir importadas, principalmente, las provenientes de China, que en gran variedad de modelos invaden el mercado interno con bajos precios. De enero a septiembre del 2018, se importaron de China 202 millones 268 mil prendas, de los cuales el $46 \%$ de esas prendas ingresó al país con un valor unitario declarado de menos de un dólar (Sociedad Nacional de Industrias, 2019).

Concretamente, el problema en cuestión puede precisarse y definirse en los siguientes términos: ¿es posible mejorar la planificación de la producción en las mypes de confecciones con un sistema informático basado en un modelo de programación matemática? 
Con el fin de responder esta pregunta, se revisaron los siguientes trabajos relacionados sobre sistemas informáticos orientados a la planificación de la producción en micro y pequeñas empresas de confecciones.

Yemane, Gebremicheal, Meraha y Hailemicheal (2020), por los problemas de baja productividad, los cuellos de botella y los largos tiempos de producción que enfrentan los fabricantes de prendas de vestir, elaboraron un software que utiliza un modelo de simulación aplicado a una empresa dedicada a la producción de pantalones. Con la aplicación del software se logró incrementar la utilización del sistema de producción alcanzando el $69 \%$, con una eficiencia de la línea del $58,42 \%$, sin que ello signifique incurrir en costos adicionales de producción.

Flores-Andrade, Guardia-Miranda, Castro-Rangel, Raymundo-Ibáñez y Pérez (2019) considerando que, por la deficiente planificación de la producción, las mypes de confecciones no podían cumplir los plazos de entrega pactados con sus clientes, lo que originaba un $80 \%$ de clientes insatisfechos, realizaron una aplicación que mejoró la producción incrementándola del $79 \%$ al $95 \%$. Para alcanzar este resultado, ellos usaron herramientas de programación lineal y programación no lineal.

Cano, Campo y Gómez (2018) resolvieron el problema de la planificación de la producción en una planta de fabricación modular. En la solución, usaron como variables la disponibilidad de personal y de maquinaria, aplicando un modelo de programación mixto basado en los resultados de un piloto de simulación discreto ejecutado en aplicaciones de MS Excel, con lo cual se logró, además, minimizar el costo por cada lote de producción.

Chen, Li, Lin y Feng (2005), tomando en cuenta que la industria de la confección es un mercado de mucha competencia y con bajas ganancias, desarrollaron un sistema de apoyo para la toma de decisiones en este tipo de industria, el cual logró minimizar los costos de producción, haciendo más atractiva la inversión. El sistema incluye tres partes principales, (i) una base de datos de entrada, (ii) una herramienta analítica y (iii) herramientas de presentación de resultados. La información de la base de datos se obtuvo de datos reales del sistema de planificación de recursos empresariales (ERP) de una empresa de confecciones. La herramienta de análisis del estudio fue el algoritmo genético (GA).

\section{METODOLOGÍA}

La metodología se fundamenta en el enfoque cuantitativo de la investigación de operaciones. Se utiliza un modelo matemático de optimización lineal por metas, bases de datos en MS Excel, por ser de uso general, para describir el problema y para resolver el modelo matemático. El $67 \%$ de las micro y pequeñas empresas manifestaron contar por lo menos con una computadora de escritorio, el $48,8 \%$ con un equipo multifuncional 
y el $35,1 \%$ con una computadora portátil (Instituto Nacional de Estadística e Informática, 2014, p. 51).

Se aplican modelos de casos de uso de UML (por sus siglas en inglés, unified modeling language, lenguaje unificado de modelado) para tener una visión clara de lo que necesitan las mypes de confecciones, así como la metodología scrum para facilitar la colaboración de los operadores.

Las interfaces para el ingreso de los datos y para los reportes de resultados se desarrollaron en Java SE.

\subsection{Modelo matemático de programación por metas o programación por objetivos} para la planificación de la producción en mypes de confecciones

\subsubsection{Definición de las variables de decisión y datos del modelo matemático}

La definición de las variables de decisión identifica cada una de las actividades en que se descompone el problema.

- Variables de decisión

$\mathrm{XPN}_{\mathrm{ij}}$ : número de unidades a producir en horario normal de las prendas modelo $\mathrm{i}=1, \ldots, \mathrm{n}$ durante el mes $\mathrm{j}=1, \ldots, \mathrm{m}$.

$\mathrm{XPE}_{\mathrm{ij}}$ : número de unidades a producir en horario extra de las prendas modelo $\mathrm{i}=$ $1, \ldots, \mathrm{n}$ durante el mes $\mathrm{j}=1, \ldots, \mathrm{m}$.

$X V{ }^{2} a_{i j}$ : número unidades vendidas de las prendas modelo $\mathrm{i}=1, \ldots, \mathrm{n}$ durante el mes $\mathrm{j}=1, \ldots, \mathrm{m}$.

$\mathrm{Xinv}_{\mathrm{ij}}$ : número en unidades en inventario final de las prendas modelo $\mathrm{i}=1, \ldots, \mathrm{n}$ durante el mes $\mathrm{j}=1, \ldots, \mathrm{m}$.

Xinvtela $a_{\mathrm{kj}}$ : tela en inventario final del diseño $k=1, \ldots, \mathrm{l}$ durante el mes $\mathrm{j}=1, \ldots, \mathrm{m}$.

Xcompra $_{\mathrm{kj}}$ : tela a comprar del diseño $\mathrm{k}=1, \ldots, \mathrm{L}$ durante el mes $\mathrm{j}=1, \ldots, \mathrm{m}$.

- Datos

C_tiempo_normal_MO $\mathrm{MO}_{\mathrm{i}}$ : costo de la mano de obra en tiempo normal de las prendas modelo $\mathrm{i}=1, \ldots, \mathrm{n}$.

C_tiempo_extra_MO $\mathrm{M}_{\mathrm{i}}$ : costo de la mano de obra en tiempo extra de las prendas modelo $\mathrm{i}=1, \ldots, \mathrm{n}$.

Pventa $a_{i}$ : de venta de las prendas modelo $\mathrm{i}=1, \ldots, \mathrm{n}$ (no lineal), depende del volumen a vender. 
C_tela $a_{\mathrm{k}}$ : costo por la compra de la materia prima $\mathrm{k}$, la cual depende del volumen de compra (no lineal).

Demanda $_{\mathrm{ij}}$ : número de unidades demandadas de las prendas modelo $\mathrm{i}=1, \ldots, \mathrm{n}$ durante el mes $\mathrm{j}=1, \ldots, \mathrm{m}$.

R_tiempo_prenda: tiempo requerido para la producción de las prendas modelo $\mathrm{i}=1, \ldots, \mathrm{n}$.

D_tiempo_normal $;$ : horas disponibles de producción en tiempo normal durante el mes $\mathrm{j}=1, \ldots, \mathrm{m}$.

D_tiempo_extraj: horas disponibles de producción en tiempo extra durante el mes $\mathrm{j}=1, \ldots, \mathrm{m}$.

R_tela_prenda: metros de tela requerida para la producción de las prendas modelo $\mathrm{i}=1, \ldots, \mathrm{n}$ (no lineal) a mayor número de prendas, menor desperdicio.

Cap_almacén_tela $:$ capacidad de almacén de tela, durante el mes j = 1,...,m.

D_tela $\mathrm{k}_{\mathrm{j}}$ : tela disponible del diseño $\mathrm{k}=1, \ldots, \mathrm{m}$, durante el mes $\mathrm{j}=1, \ldots, \mathrm{m}$.

Cap_almacén_prendas j: capacidad de almacén durante el mes j = 1,..,m.

\subsubsection{Sistema de restricciones del modelo}

Para la construcción del sistema de restricción es necesario considerar que existe una limitación cuantitativa.

a) Balance de producción e inventario de productos terminados: el inventario final de la prenda modelo $i$, durante el mes $j$, es igual al inventario inicial de la prenda $i$ durante el mes $j$, más la producción de la prenda $i$ durante el mes $j$, menos la venta de la prenda modelo $i$ durante el mes $j$.

$$
\begin{gathered}
\mathrm{Xinv}_{\mathrm{ij}}=\mathrm{Xinv}_{\mathrm{ij}-1}+\mathrm{XPN}_{\mathrm{ij}}+\mathrm{XPE}_{\mathrm{ij}}-\mathrm{XVenta}_{\mathrm{ij}} \\
\forall \mathrm{i}=1, \ldots, \mathrm{n} ; \quad \forall \mathrm{j}=1, \ldots, \mathrm{m}
\end{gathered}
$$

b) Balance de inventario de tela: el inventario final de tela diseño $k$, durante el mes $j$, es igual al inventario inicial de la tela diseño $k$ durante el mes $j$, más el requerimiento de la tela para la prenda modelo $i$, por el total de prendas a producir del modelo $i$, tanto en tiempo normal y tiempo extra durante el mes $j$.

$$
\begin{gathered}
\text { XInvtela }_{\mathrm{kj}}=\text { XInvtela }_{\mathrm{kj}-1}+ \\
\sum_{\mathrm{i}=1}^{\mathrm{n}} \text { Rtelaprenda }_{\mathrm{i}} *\left(\mathrm{XPN}_{\mathrm{ij}}+\mathrm{XPE}_{\mathrm{ij}}\right)-\text { XVenta }_{\mathrm{ij}} ; \\
\forall \mathrm{k}=1 \ldots \mathrm{l} ; \quad \forall \mathrm{j}=1, \ldots, \mathrm{m}
\end{gathered}
$$


c) Satisfacción de la demanda: representa, en el modelo matemático, las prendas a vender de acuerdo con los pedidos de la prenda modelo $i$, durante el mes $j$; el valor que tome esta variable en la solución no puede sobrepasar la demanda de la prenda modelo $i$, durante el mes $j$.

$$
\text { XVenta }_{\mathrm{ij}} \leq \text { Demanda }_{\mathrm{ij}} ; \quad \forall \mathrm{i}=1, \ldots, \mathrm{n} ; \quad \forall \mathrm{j}=1, \ldots, \mathrm{m}
$$

d) Capacidad de inventario de productos terminados: el total de prendas a almacenar en un mes no puede sobrepasar la capacidad del almacén de productos terminados.

$$
\sum_{\mathrm{i}=1}^{\mathrm{n}} \mathrm{XInv}_{\mathrm{ij}} \leq \text { Cap_almacén_prenda }_{\mathrm{ij}} ; \quad \forall \mathrm{j}=1, \ldots, \mathrm{m}
$$

e) Capacidad de inventario de telas: el total de tela a almacenar en un mes no puede sobrepasar la capacidad del almacén.

$$
\sum_{\mathrm{i}=1}^{\mathrm{n}} \text { XInvtela }_{\mathrm{kj}} \leq \text { Cap_almacén_tela }_{\mathrm{k}} ; \quad \forall \mathrm{k}=1, \ldots, \mathrm{l}
$$

f) Disponibilidad del tiempo normal en el mes $j$ : el tiempo normal de producción usado durante el mes $j$ no debe sobrepasar la disponibilidad de tiempo normal en el mes $j$.

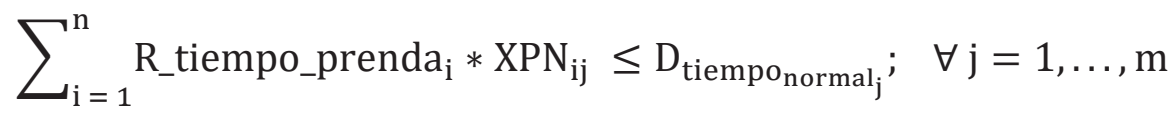

g) Disponibilidad de tiempo de producción extra en el mes j: el tiempo total extra de producción no puede sobrepasar la disponibilidad de tiempo extra.

$$
\sum_{\mathrm{i}=1}^{\mathrm{n}} \mathrm{R}_{-} \text {tiempo_prenda } \mathrm{i} * \mathrm{XPR}_{\mathrm{ij}} \leq \mathrm{D}_{\text {tiempo }_{\text {extra }}} ; \quad \forall \mathrm{j}=1, \ldots, \mathrm{m}
$$

h) Disponibilidad de tela: cada tela tiene un diseño y un color definido, todas las prendas a producir tienen un requerimiento de tela y no podemos usar más tela de la que nos pueden vender los proveedores.

$$
\begin{gathered}
\sum_{\mathrm{i}=1}^{\mathrm{n}} \text { Reqtela }_{\mathrm{ik}} *\left(\mathrm{XPN}_{\mathrm{ij}}+\mathrm{XPE}_{\mathrm{ij}}\right)<=\text { D_tela }_{\mathrm{kj}} \\
\forall \mathrm{k}=1, \ldots, \mathrm{l} ; \quad \forall \mathrm{i}=1, \ldots, \mathrm{n}
\end{gathered}
$$




\subsubsection{Sistema de restricciones de metas del modelo}

a) Meta 1, costo de la mano de obra: no sobrepasar el presupuesto asignado para el pago al personal mensualmente.

$$
\sum_{\mathrm{j}=1}^{\mathrm{n}} \mathrm{C}_{\mathrm{j}} * \mathrm{X}_{\mathrm{j}}
$$

$=$ presupuesto asignado mensual costo mano obra $+\mathrm{e}_{1}-\mathrm{d}_{1}$

b) Meta 2, presupuesto de compra de tela: no sobrepasar el presupuesto asignado para la compra de la tela mensualmente.

$$
\begin{gathered}
\sum_{\mathrm{k}=1}^{\mathrm{l}} \sum_{\mathrm{j}=1}^{\mathrm{m}} \text { C_tela }_{\mathrm{k}} * \text { XCompra }_{\mathrm{kj}} \\
=\text { presupuesto asignado mensual compra tela }+\mathrm{e}_{2}-\mathrm{d}_{2}
\end{gathered}
$$

c) Meta 3, ingresos por vender: lograr un ingreso mínimo esperado por vender las prendas.

$$
\begin{aligned}
& \sum_{\mathrm{i}=1}^{\mathrm{n}} \sum_{\mathrm{j}=1}^{\mathrm{m}} \operatorname{Pventa}_{\mathrm{i}} *\left(\mathrm{XPN}_{\mathrm{ij}}+\mathrm{XPE}_{\mathrm{ij}}\right) \\
& =\text { mínimo ingreso mensual }+\mathrm{e}_{3}-\mathrm{d}_{3}
\end{aligned}
$$

d) Meta 4, satisfacción de la demanda: satisfacer la demanda de la prenda modelo $i$ durante el mes $j$.

$$
\mathrm{XVenta}_{\mathrm{ij}}=\text { Demanda }_{\mathrm{ij}}+\mathrm{e}_{\mathrm{ij}}-\mathrm{d}_{\mathrm{ij}} ; \quad \forall \mathrm{i}=1 \ldots \mathrm{n} ; \quad \forall \mathrm{j}=1, \ldots, \mathrm{m}
$$

e) Meta 5, máximo de horas extras: el total de horas extras mensuales no debe sobrepasar del valor permitido por la ley.

$$
\begin{gathered}
\sum_{\mathrm{i}=1}^{\mathrm{n}} \text { R_tiempo_prenda }_{\mathrm{i}} * \mathrm{XPE}_{\mathrm{ij}}=\text { valor permitido } \\
+\mathrm{e}_{5}-\mathrm{d}_{5} ; \quad \forall \mathrm{j}=1, \ldots, \mathrm{m}
\end{gathered}
$$




\subsubsection{Sistema de restricciones de no negatividad}

$$
\begin{gathered}
\mathrm{XPN}_{\mathrm{ij}}>=0 ; \forall \mathrm{i}=1 \ldots \mathrm{n} ; \forall \mathrm{j}=1, \ldots, \mathrm{m} \\
\mathrm{XPE}_{\mathrm{ij}}>=0 ; \forall \mathrm{i}=1 \ldots \mathrm{n} ; \forall \mathrm{j}=1, \ldots, \mathrm{m} \\
\mathrm{XVenta}_{\mathrm{ij}}>=0 ; \forall \mathrm{i}=1 . . \mathrm{n} ; \forall \mathrm{j}=1, \ldots, \mathrm{m} \\
\mathrm{Xinv}_{\mathrm{ij}}>=0 ; \forall \mathrm{i}=1 . . \mathrm{n} ; \forall \mathrm{j}=1, \ldots, \mathrm{m} \\
\text { Xinvtela }_{\mathrm{kj}} \geq 0 ; \forall \mathrm{k}=1 . . \mathrm{l} ; \forall \mathrm{j}=1, \ldots, \mathrm{m} \\
\text { XCompra }_{\mathrm{kj}} \geq 0 ; \forall \mathrm{k}=1 . . \mathrm{l} ; \forall \mathrm{j}=1, \ldots, \mathrm{m}
\end{gathered}
$$

\subsubsection{Definición de la función objetivo}

La función objetivo representa la suma de las desviaciones de las metas propuestas.

$$
\text { Minimizar } Z: e_{1}+e_{2}+d_{3}+d_{4 K}+e_{5}
$$

\subsection{Requerimientos para el desarrollo del sistema informático para la planificación de la producción}

Los requerimientos para el desarrollo del sistema informático para la planificación de la producción en mypes de confecciones están divididos en dos grupos: los requerimientos para el modelo de programación matemática de la planificación de la producción y los requerimientos de software.

2.2.1 Requerimientos para el desarrollo del modelo de programación matemática de la planificación de la producción

La información obtenida de las mypes analizadas indican los siguientes factores del proceso productivo a considerar en el sistema informático para la planificación de la producción:

- Tiempo estándar de una operación, obtenido de la hoja de registro de tiempo o métodos de control de tiempo.

- Los requerimientos promedios de materia prima, disponibilidad de horas hombre en tiempo normal, disponibilidad de horas hombre en tiempo extra, disponibilidad de materias primas, costos de la mano de obra directa, costo de la materia prima, pedidos de las prendas y los precios de venta. Esta información fue obtenida de la hoja de registro de datos de la empresa. 
Los datos de entrada del modelo de programación matemática para la planificación se ordenan en un modelo de gestión del área de producción, como se ilustra a continuación:

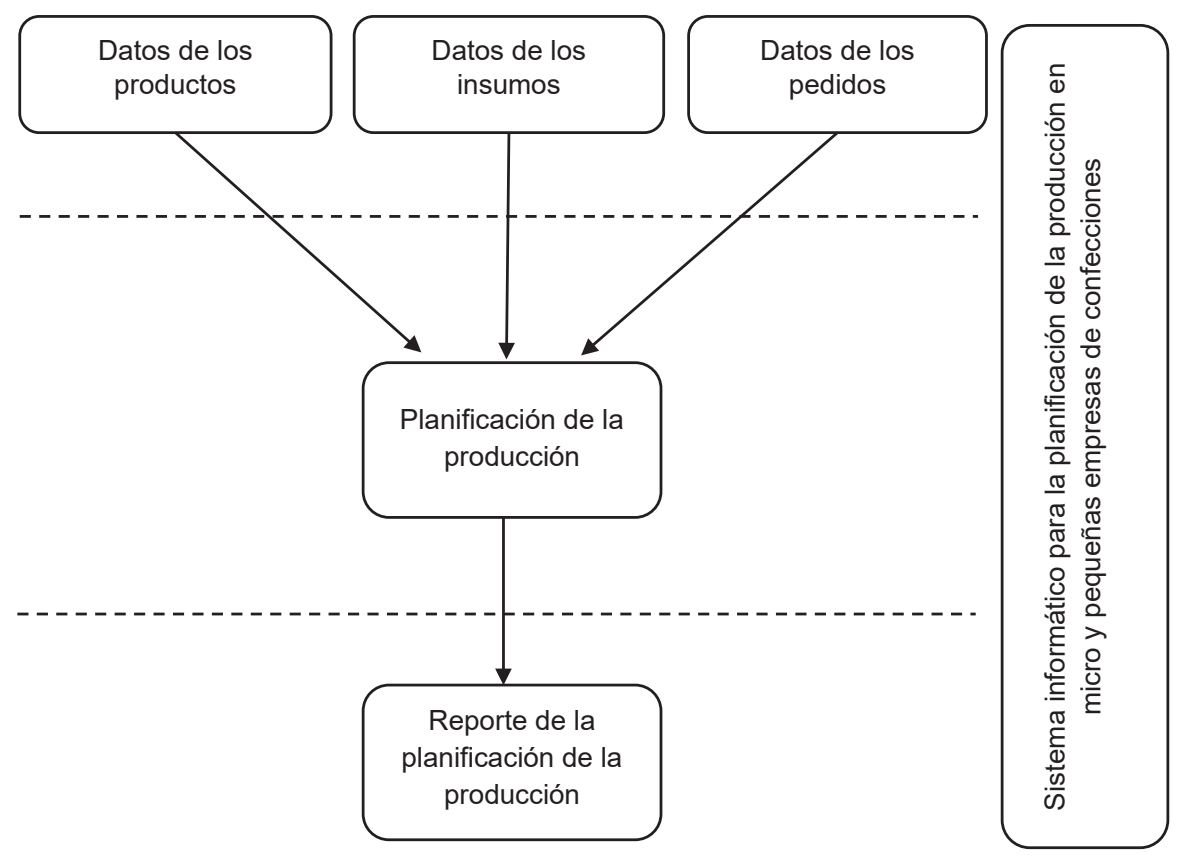

Figura 1. Modelo de gestión del área de producción en mypes de confecciones

\section{Elaboración propia}

En base al modelo de gestión del área de producción, se desarrolló el sistema informático para la planificación de la producción en mypes de confecciones, el cual se compone de las siguientes interfaces:

- Ingreso de los datos de los productos y los datos del insumo.

- Ingreso de los datos de los pedidos.

- Ver pedidos.

- Reporte de la planificación de la producción.

2.2.2 Requerimientos del software del sistema informático para la planificación de la producción

A la fecha, la mayoría de las mypes de confecciones contaban con una computadora con el sistema operativo Windows y Office. Esto favoreció la elección de esta plataforma, a fin de minimizar la inversión, y a que los usuarios estuvieran familiarizados con MS Excel. 
Las interfaces para el ingreso de los datos y para el reporte de resultados se desarrollaron en la plataforma Java SE (Standar Edtion) JDK13 UPTE, plataforma NetBeans IDE 7.2.1; sin embargo, no fue necesario la instalación de este software en las computadoras, ya que solo se requería el ejecutable.

2.2.2.1 Diagrama de clases del sistema informático para la planificación de la producción El siguiente diagrama describe la estructura del sistema, mostrando las clases, los atributos y las relaciones entre ellos:

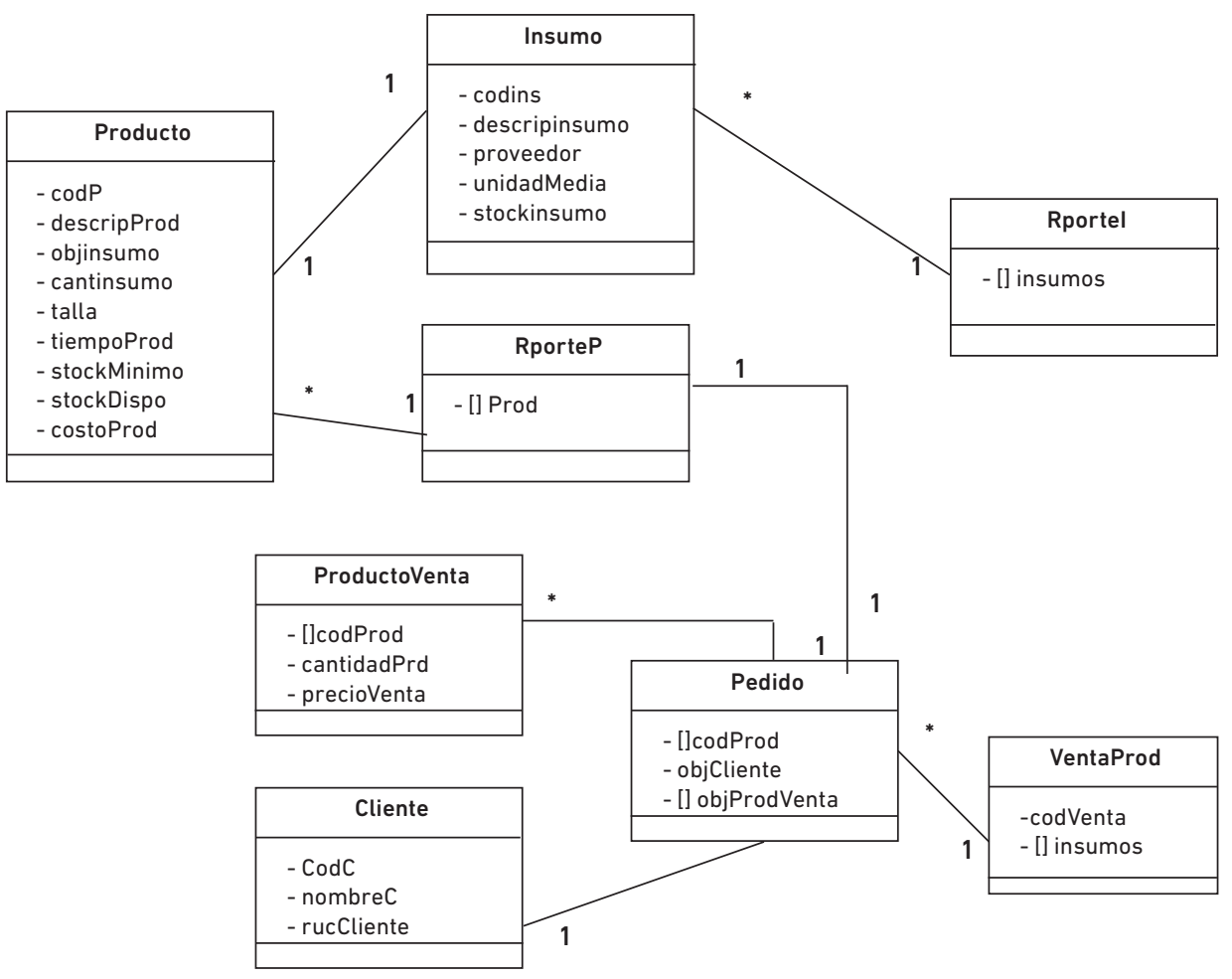

Figura 2. Diagrama de clases

Elaboración propia

2.2.2.2 Análisis de requerimientos de los casos de uso

Los modelos de casos de uso de UML se usan para tener una visión clara de lo que necesitan las mypes de confecciones que han sido evaluadas.

El siguiente diagrama describe el caso de uso que se ha desarrollado usando el software Rational Rose Modeler. 


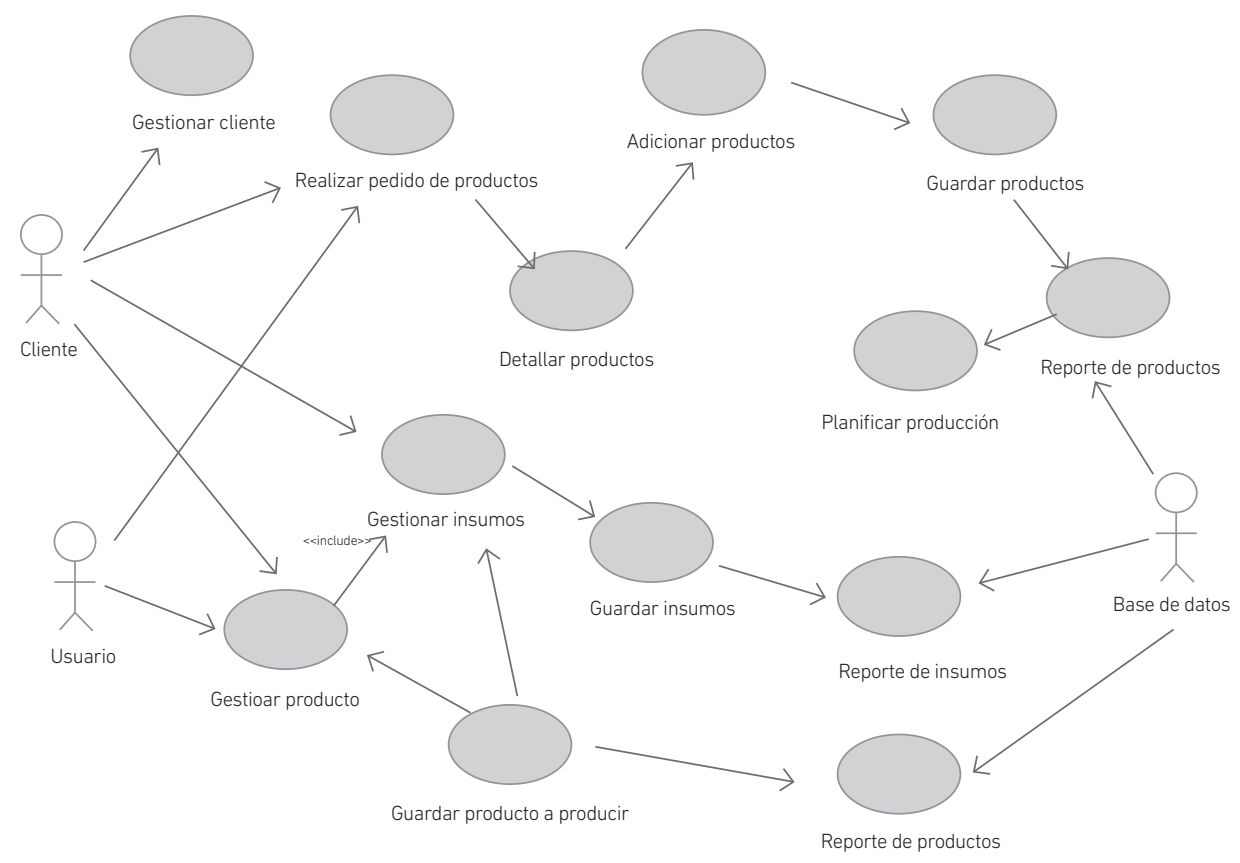

Figura 3. Casos de uso del sistema informático para el planeamiento de la producción en mypes de confecciones

Elaboración propia

\subsubsection{Diseño de la base de datos}

Para el diseño de la base de datos se aplicaron las técnicas de normalización de los datos. Se diseñaron las siguientes bases de datos: productos, telas, clientes y pedidos.

2.2.2.4 Diseño, desarrollo y pruebas del sistema informático para la planificación de la producción, versión 1

La interfaz principal contiene cuatro interfaces: "Abrir", "Ver pedidos", "Reporte de planificación" y "Acerca de", como se muestra a continuación.

En la opción "Abrir" se tienen dos alternativas: "Ingresar producto" e "Ingreso de pedidos".

- Ingresar producto: información de los productos, así como los datos del insumo.

- Ingreso de pedidos: datos del cliente, como el código asignado por el cual se le reconoce en el sistema, la razón social y RUC, y la lista de los productos de cada pedido. 
En la opción "Ver pedidos" se genera el consolidado de todos los pedidos, por código de producto, de la cantidad total, esto es importante porque diversos clientes pueden pedir el mismo producto.

En la opción "Reporte de planificación" se genera el reporte de salida, lo cual indica la cantidad que se debe producir de cada producto.

\section{RESULTADOS}

El sistema informático para la planificación de la producción se instaló en dos mypes de confecciones de la ciudad de Lima en el año 2019 y quedó pendiente en hacerlo en otras dos, debido a que no fue posible por las cuarentenas y restricciones causadas a nivel global por la pandemia de la COVID-19.

\subsection{Mype de confección de camisas para caballeros}

La primera mype se dedica a la confección de camisas para caballeros, en distintos modelos, tanto de vestir como casual. En ella laboran seis trabajadores en el área de producción.

Antes de la instalación del sistema informático, la empresa solo se esforzaba en cumplir con todos los pedidos, en mantener al personal ocupado y a las máquinas produciendo el mayor tiempo posible.

Se ha demostrado que se puede aprovechar toda la capacidad de producción, confeccionando únicamente las prendas que generan altos niveles de productividad.

Respecto al indicador, el sistema informático para la planificación de la producción en mypes de confecciones se replicó desde el mes de enero hasta noviembre del 2019, obteniéndose los resultados de la figura 4. 


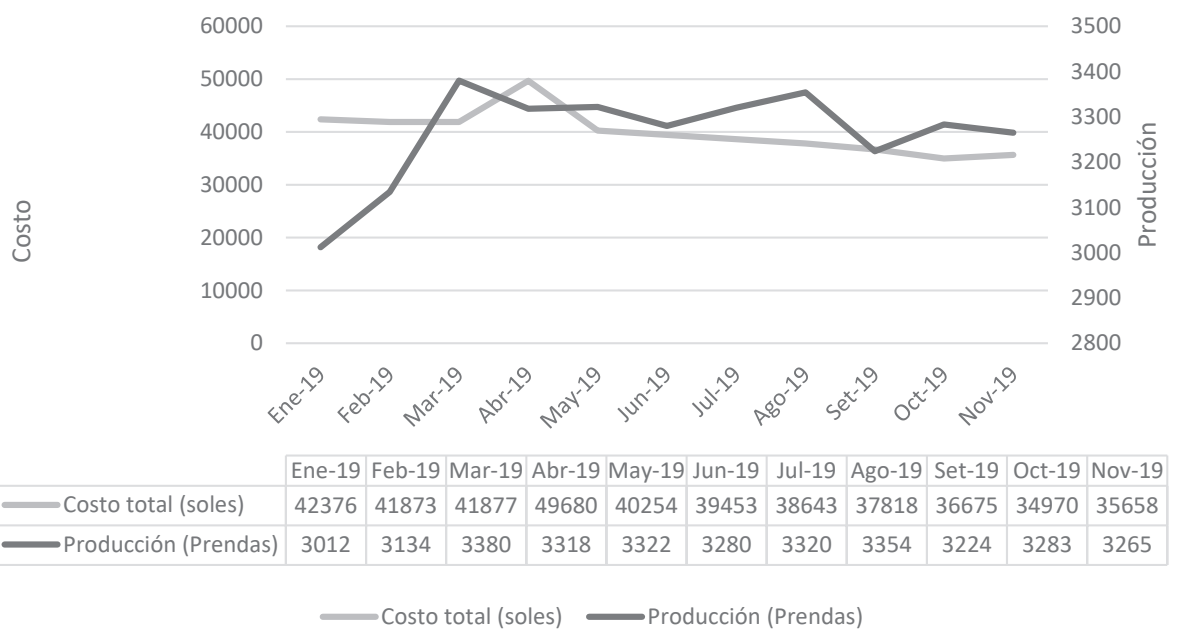

Figura 4. Gráfica de la producción y los costos desde enero hasta noviembre del 2019 en la mype de confección de camisas para caballeros

Elaboración propia

Al hacer la comparación del indicador de la productividad de la empresa, antes y después de usar el sistema informático para la planificación de la producción en mypes de confecciones, de enero a noviembre del 2018 (sin el sistema informático) y de enero a noviembre del 2019 (con el sistema informático), se reflejaron las diferencias representadas en la figura 5.

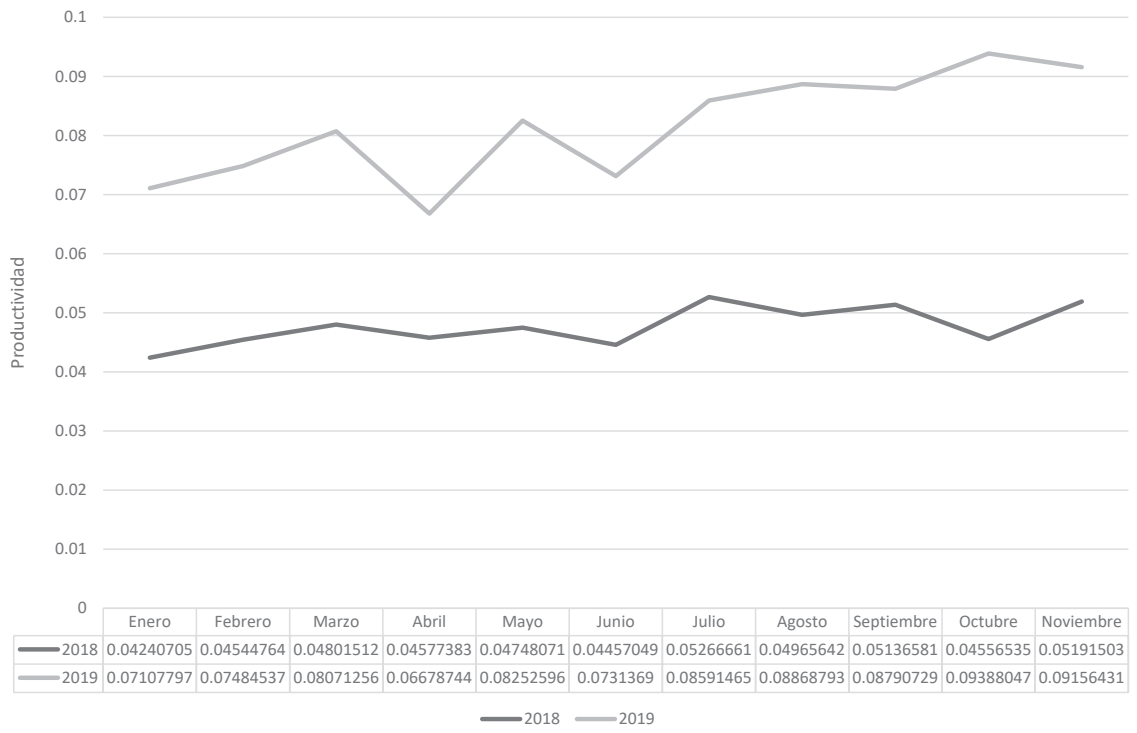

Figura 5. Gráfica de la comparación de la productividad desde enero hasta noviembre de los años 2018 y 2019 en la mype de confección de camisas para caballeros

Elaboración propia 
Cuando se compara la productividad de enero a noviembre del 2018 con el mismo período del 2019, se observa un incremento en los meses de análisis, lo que refleja la mejora del indicador al instalar el sistema informático para la planificación de la producción.

\subsection{Mype de confección de polos unisex para adultos}

La segunda mype se dedica a la confección de polos unisex para adultos. Antes de la instalación del sistema informático, la empresa, con el propósito de satisfacer los pedidos, trabajaba hasta los domingos.

Respecto al indicador, el sistema informático para la planificación de la producción en mypes de confecciones, se replicó en esta empresa desde el mes de enero hasta diciembre del 2019, obteniéndose los resultados de la figura 6.

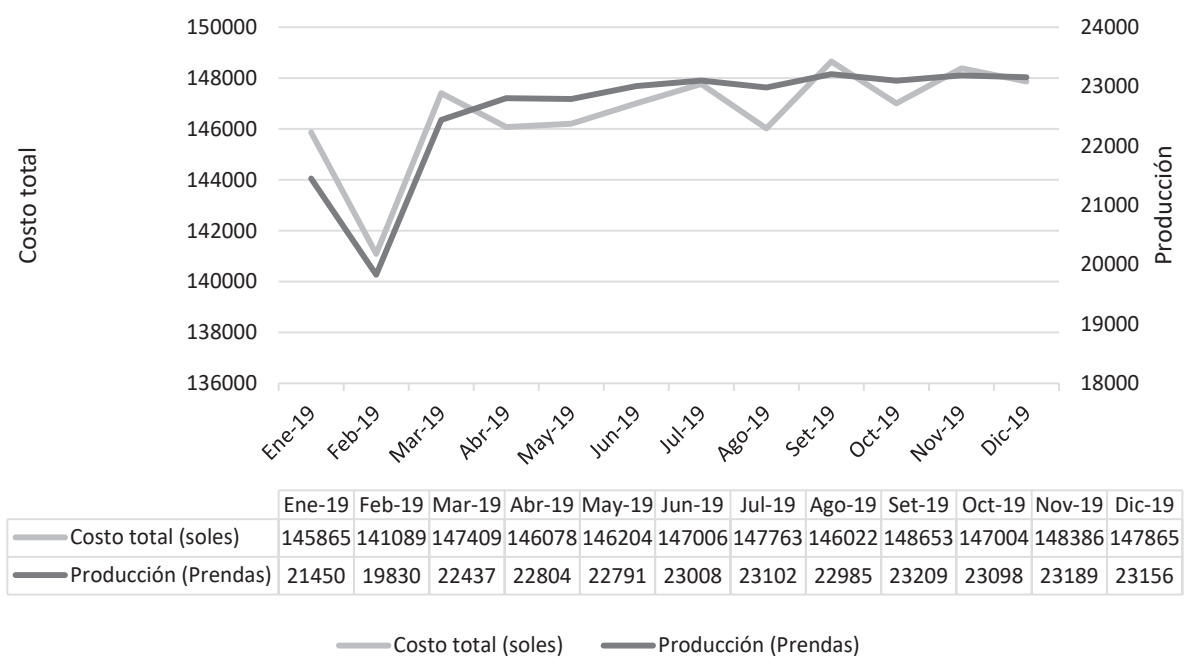

Figura 6. Gráfica de la producción y costos desde enero hasta diciembre del 2019 en la mype de confección de polos unisex para adultos

Elaboración propia 
Al hacer la comparación del indicador de la productividad de la empresa de confección de polos, antes y después de usar el sistema informático para la planificación, de enero a diciembre del 2018 (sin el sistema informático) y de enero a diciembre del 2019 (con el sistema informático), se lograron graficar los resultados en la figura 7.

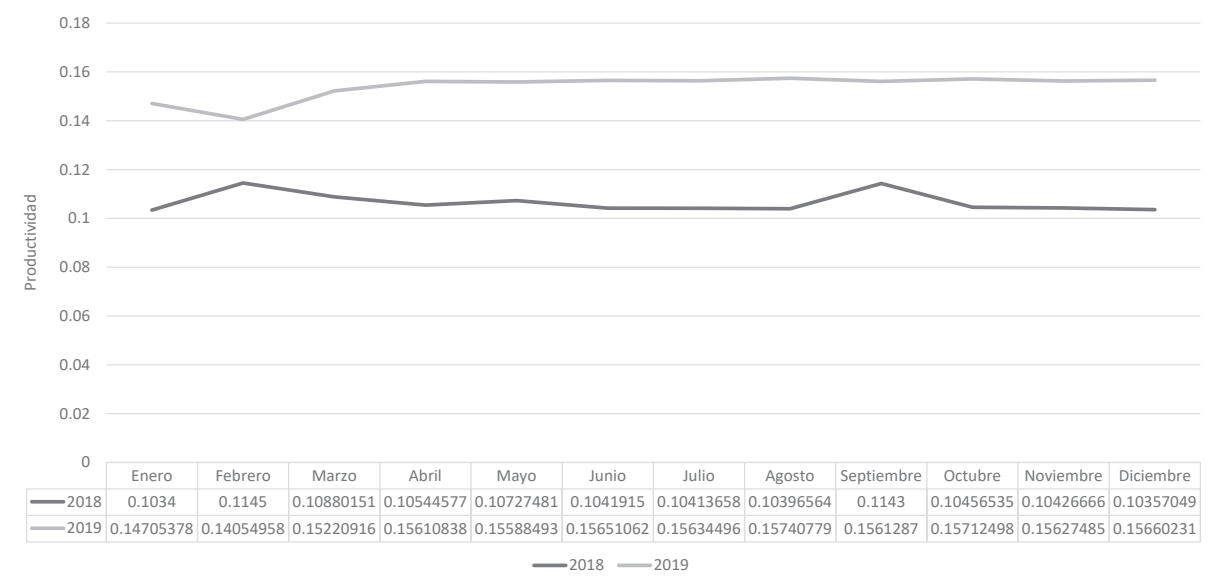

Figura 7. Gráfica de la comparación de la productividad desde enero hasta diciembre de los años 2018 y 2019 en la mype de confección de polos unisex para adultos

Elaboración propia

Cuando se compara la productividad de enero a diciembre del 2018 con los mismos meses del año 2019, se logra demostrar que la productividad se incrementó en todo el período de análisis, lo que indica que sí existe una mejora después de la implementación del sistema informático para la planificación de la producción.

Cabe resaltar que se evidenció una gran aceptación del sistema informático mencionado por parte de las personas asignadas para su implementación, debido a que les resultaba fácil por la familiaridad que tenían con el uso del MS Excel. Igualmente, hicieron énfasis en que dicho sistema les permitía tomar una mejor decisión al momento de aceptar un nuevo pedido.

\section{DISCUSIÓN}

Con el sistema informático para la planificación de la producción en mypes de confección, la variación de productividad promedio de los once meses de análisis en la primera mype (confección de camisas para caballeros) fue del 70,86 \%, en la segunda mype (confección de polos unisex para adultos) la variación de productividad promedio de los doce meses de análisis fue del 44,83 \%, sin incurrir en costos adicionales por la implementación del software en MS Excel. Por el contrario, Yemane et al. (2020), 
con el fin de incrementar la productividad proponen un sistema utilizando un modelo de simulación con el software ARENA, aplicado a una empresa dedicada a la producción de pantalones, logrando incrementar la utilización del sistema a 69 \% con una eficiencia de la línea del $58,42 \%$, pero para el caso que nos ocupa debía invertirse en la adquisición del software ARENA.

El sistema informático para la planificación de la producción en mypes de confecciones utilizó un modelo de programación matemática de optimización lineal por metas. En cambio, Ghasemy Yaghin (2020) adaptó un procedimiento de solución analítica eficiente para convertir el modelo de programación original altamente no lineal en un equivalente de programación convexo, porque se presentaba el problema de planificación de la producción agregada de varios productos, períodos y canales de venta; los resultados de la investigación indicaron una relación positiva entre la constante de escala de la demanda dependiente del precio y la tasa de beneficio total.

\section{CONCLUSIONES}

Las conclusiones que se obtuvieron fueron las siguientes:

- El sistema informático para la planificación de la producción en mypes de confección, al ser desarrollado en el entorno MS Excel, software de uso común en las mypes de confecciones, no incurre en costos adicionales para su implementación.

- El uso de metodologías ágiles, en este caso, scrum, facilita la participación y colaboración activa de los usuarios.

- La aplicación del sistema informático para la planificación de la producción en mypes de confección, basada en un modelo de programación matemático y la metodología scrum, logró incrementar la productividad, permitiendo alcanzar los niveles de producción esperados, durante el período de análisis.

- El desarrollo de un sistema informático para la planificación de la producción en mypes de confección es viable siguiendo los principios de la metodología scrum, permitiendo adaptar el sistema según el tipo de prenda a confeccionar.

- El desarrollo del sistema informático para la planificación de la producción en mypes de confección fue aceptado favorablemente en las dos mypes donde se instaló debido a su fácil uso y su rápida adaptación a las necesidades de estas. La ocurrencia de la pandemia no permitió la instalación en otras empresas.

- El proceso productivo en mypes de confecciones tiene un modelo estándar, lo que permite implementarlo en cualquier tipo de mype de confecciones. 


\section{REFERENCIAS}

Camero, I. y Vargas, E. (2012). Planeación, programación y control de la producción en la pyme de confecciones Verney Jiménez E. U. en Bogotá (tesis de grado). Universidad Libre, Bogotá, Colombia.

Cano, J. A., Campo, E. A., y Gómez, R. A. (2018). Simulación de eventos discretos en la planificación de producción para sistemas de confección modular (tesis de grado). Universidad de Medellín, Colombia.

Chen, R., Li, S., Lin, C., y Feng, C. (13-15 de octubre del 2005). A GA-based global decision support system for garment production. In International Conference on Neural Networks and Brain (pp. 805-809). Beijing, China. https://ieeexplore.ieee.org/ document/1614746

Cuatro de cada cinco pymes son informales: ¿Es suficiente reducir el IGV? ( 25 de agosto de 2016). Gestión. Recuperado de https://gestion.pe/economia/cuatro-cincopymes-son-informales-suficiente-reducir-igv-147423-noticia/

Eppen, G. D., Gould, F. J., Schmidt, C. P., Moore, J. H., y Weatherford, L. R. (2000). Investigación de operaciones en la ciencia administrativa. Construcción de modelos para la toma de decisiones con hojas de cálculo electrónicas. México D. F.: Pearson.

Flores-Andrade, K., Guardia-Miranda, R., Castro-Rangel, P., Raymundo-lbáñez, C., y Pérez, M. (2019). Production management model through MPS and line balancing to reduce the non-fulfillment of orders in lingerie clothing MSEs in Peru. IOP Conference Series: Materials Science and Engineering. Banda Aceh, Indonesia.

Ghasemy Yaghin, R. (2020). Enhancing supply chain production-marketing planning with geometric multivariate demand function (case study of textile industry). Computers \& Industrial Engineering, 140. https://doi.org/10.1016/j.cie.2019.106220

Hillier, F. S., y Lieberman, G. J. (2010). Introducción a la investigación de operaciones

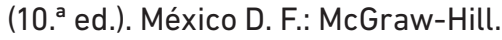

Instituto Nacional de Estadística e Informática. (2014). Encuesta de micro y pequeña empresa, 2013. Recuperado de https://www.inei.gob.pe/media/MenuRecursivo/ publicaciones_digitales/Est/Lib1139/libro.pdf

Instituto Nacional de Estadística e Informática. (2011). Encuesta de micro y pequeña empresa, 2010. Recuperado de https://www.inei.gob.pe/media/MenuRecursivo/ publicaciones_digitales/Est/Lib0980/Libro.pdf

Render, B., Stair, R. M., y Hanna, M. E. (2016). Métodos cuantitativos para los negocios. México D. F.: Pearson Educación. 
Sociedad Nacional de Industrias. (2019). Revista Institucional Industria Peruana, (933). Recuperado de https://www.sni.org.pe/wp-content/uploads/2019/05/Revistadic-2018-ener-2019-933-1.pdf

Taha, H. A. (2012). Investigación de operaciones (9. a ed.). México D. F.: Pearson Educación.

Winston, W. L., y Goldberg, J. B. (2005). Investigación de operaciones: Aplicaciones y algoritmos. México D. F.: Thomson.

Yang, L. N. (abril de 2012). Research and development of the generation method of clothing process diagram based on CAPP integration system. Advanced Materials Research, 503-504, 182-185. https://doi.org/10.4028/www.scientific. net/amr.503-504.182

Yemane, A., Gebremicheal, G., Meraha, T., y Hailemicheal, M. (2020). Productivity improvement through line balancing by using simulation modeling (case study Almeda Garment Factory). Journal of Optimization in Industrial Engineering, 13(1), 153-165. doi:10.22094/JOIE.2019.567816.1565 